\title{
Body Mass Index at age 25 and All-cause Mortality in Whites and African Americans: The Atherosclerosis Risk in Communities Study
}

\author{
June Stevens, MS, $\mathrm{PhD}^{1,2}$, Kimberly $\mathbf{P}$ Truesdale, $\mathrm{PhD}^{1}$, Chin-Hua Wang, $\mathrm{PhD}^{1}$, Jianwen \\ Cai, $\mathrm{PhD}^{3}$, and Eva Erber, $\mathbf{M S}^{1}$ \\ ${ }^{1}$ Department of Nutrition, Gillings School of Global Public Health, University of North Carolina at \\ Chapel Hill, Chapel Hill, NC, USA \\ ${ }^{2}$ Department of Epidemiology, Gillings School of Global Public Health, University of North \\ Carolina at Chapel Hill, Chapel Hill, NC, USA \\ ${ }^{3}$ Department of Biostatistics, Gillings School of Global Public Health, University of North Carolina \\ at Chapel Hill, Chapel Hill, NC, USA
}

\begin{abstract}
Purpose-Approximately $20 \%$ of young adults in the United States are obese, and most gain weight between young and middle adulthood. Few studies have examined the association between elevated BMI in early adulthood and mortality or examined such effects independent of changes in weight. We know of no studies in African American samples.
\end{abstract}

Methods-We used data from 13,941 African American and White adults who self-reported their weight at age 25 and had weight and height measured when they were 45-64 years of age (1987-89). Date of death was ascertained from 1987 to 2005. Hazard ratios and hazard differences for the effects of BMI at age 25 on all-cause mortality were determined using Cox proportional hazard and additive hazard models, respectively.

Results-In the combined ethnic-gender groups, the hazard ratio associated with a $5 \mathrm{~kg} / \mathrm{m}^{2}$ increment in BMI at age 25 was 1.28 (95\% CI: 1.22, 1.35) and the hazard difference was 2.75 $(2.01,3.50)$ deaths/1,000 person-years. Associations were observed in all four ethnic-gender groups. Models including weight change from age 25 to age in 1987-89 resulted in null estimates for BMI in African American men, while associations were maintained or only mildly attenuated in other ethnic-gender groups.

Conclusions-Excess weight during young adulthood should be avoided as it contributes to increases in death rates that may be independent of changes in weight experienced in later life. Further study is needed to better understand these associations in African American men.

\section{Keywords}

BMI; young adulthood; mortality; White Americans; African Americans; risk ratio; risk difference

(C) 2011 Society for Adolescent Medicine. Published by Elsevier Inc. All rights reserved.

Corresponding author: Dr. June Stevens, American Institute for Cancer Research Distinguished Professor, Chair of the Department of Nutrition, 245 Rosenau Hall, CB 7461, Gillings School of Global Public Health, University of North Carolina at Chapel Hill, Chapel Hill, NC 27599, USA. June_Stevens@unc.edu; Phone: 919-966-7218; Fax: 919-966-7215.

Publisher's Disclaimer: This is a PDF file of an unedited manuscript that has been accepted for publication. As a service to our customers we are providing this early version of the manuscript. The manuscript will undergo copyediting, typesetting, and review of the resulting proof before it is published in its final citable form. Please note that during the production process errors may be discovered which could affect the content, and all legal disclaimers that apply to the journal pertain. 


\section{INTRODUCTION}

Between 1988 and 2004, obesity prevalence in young adults, aged 18-29, increased by almost $100 \%[1,2]$. Over the past 30 years prevalence rates of obesity almost tripled in young men (ages 20-39) and more than tripled in young women [3,4]. Obesity rates in young African Americans are even higher than those observed in the general population. In NHANES 2007-2008 prevalence rates of obesity in 20-39 year old non-Hispanic African Americans compared to Whites were $34.7 \%$ versus $26.3 \%$ in men and $47.2 \%$ versus $31.3 \%$ in women [4].

There is reason for concern that these high rates of obesity in young adults could signal future declines in the health and vitality of the American population. We know of four studies [5-8] that have examined the effect of body weight in early adulthood (18-22 years) on all-cause mortality among predominantly White populations. All these studies showed a positive association between body weight and all-cause mortality with risks ranging from $16 \%$ to $95 \%$ comparing the highest BMI categories (ranging from $\geq 24.2$ to $\geq 26.0 \mathrm{~kg} / \mathrm{m}^{2}$ ) to a lower category (ranging from $<18.5$ to $<25.0 \mathrm{~kg} / \mathrm{m}^{2}$ ) in multivariate adjusted models. To our knowledge, two studies have examined the impact of BMI during early adulthood on mortality in Japanese $[9,10]$. Recalled information on BMI at age 20 was collected as part of the Ohsaki National Health Insurance study of 30,080 men and women 40-79 years of age in 1994 [9]. Multivariable adjusted hazard ratios (HR) were 1.13 (95\% CI: 1.03-1.24) for a BMI of $25.0-29.9 \mathrm{~kg} / \mathrm{m}^{2}$ and 1.39 (95\% CI: $1.11-1.75$ ) for a BMI of $>30.0 \mathrm{~kg} / \mathrm{m}^{2}$. The second study used self-reported weight at age 25 from 8,006 Japanese American men 45-68 years old in 1939-1945 who were participants in the Honolulu Heart Study [10]. Mortality rates were higher among men with a BMI $>26.3 \mathrm{~kg} / \mathrm{m}^{2}$ at age $25(14.1$ per 1,000$)$ compared to men with a BMI $<21.2 \mathrm{~kg} / \mathrm{m}^{2}$ (7.1 per 1,000).

None of the available studies examined the contribution of early obesity among African Americans or adjusted for the effects of subsequent weight change. The purpose of this paper is to estimate the associations of BMI at age 25 to all-cause mortality in African Americans and Whites. In addition, we will test whether adjusting for subsequent changes in BMI between age 25 and middle adulthood (ages 45-64) alters the impact of BMI in young adulthood on mortality in both ethnic groups.

\section{METHODS}

\section{Study population}

The Atherosclerosis Risk in Communities (ARIC) study is a prospective investigation of the natural history and etiology of atherosclerosis and cardiovascular disease in four US communities: Forsyth County, NC; Jackson, MS; the northwestern suburbs of Minneapolis, MN; and Washington County, MD [11]. Baseline data were collected in 1987-1989 on 15,792 White and African American adults 45-64 years old. Three additional examinations occurred at approximately three year intervals. This study was approved by the Institutional Review Boards (IRB) at each field center and this analysis was approved by the University of North Carolina at Chapel Hill Public Health and Nursing IRB on research involving human subjects.

\section{Measures and covariates}

At visit 1, participants were asked to recall their body weight at age 25 . Interviewers used time-associated life events to assist participants in their recall. Body weight was measured at all examinations in a scrub suit to the nearest pound using a beam balance scale. Height 
(without shoes) was measured to the nearest centimeter using a metal ruler attached to a wall and a standard triangular headboard. BMI (weight $(\mathrm{kg}) /$ height $\left(\mathrm{m}^{2}\right)$ ) was calculated at age 25 and at visit 1 using visit 1 height. Absolute weight change was calculated as the difference in self-reported weight at age 25 and measured weight at visit 1.

To ascertain deaths, the ARIC study used cohort and community surveillance including surveying discharge lists from local hospitals and local obituaries and conducting annual reviews of vital statistics tapes to detect additional deaths. Death certificates were obtained for all deaths. All-cause mortality was defined as death during the period between 1987 and December 31, 2005. Follow-up years were calculated as years from visit 1 to date of death, loss to follow-up, or December 31, 2005. Less than 1\% of the cohort was lost to follow-up.

Date of birth, race/ethnicity and gender were self-reported during the recruitment phase and confirmed during the first examination. Covariates assessed by interviewer administered questionnaires at visit 1 included education, cigarette smoking status, alcohol consumption and physical activity. We categorized education as less than a high school education, high school graduate or at least some college. At visit 1, participants were asked "have you ever smoked cigarettes", "how old were you when you first started regular cigarette smoking", "do you now smoke cigarettes" and "how old were you when you stopped". Using the data generated, we categorized participants' cigarette smoking status at age 25 (smoker or nonsmoker) and at visit 1 (current, former or never cigarette smokers). Participants were asked whether they "presently drink alcoholic beverages", "ever consumed alcoholic beverages" and the quantity of drinks (wine, beer, hard liquor) they usually consume per week. The amount of ethanol consumed (in grams per week (g/wk)) was calculated assuming the following: $4 \mathrm{oz}$ wine $=10.8 \mathrm{~g}, 12 \mathrm{oz}$ beer=13.2 g, $1.5 \mathrm{oz}$ hard liquor=15.1 g. For a drinker who reported less than one drink per week, the alcohol consumption was recorded as zero grams per week. At visit 1, participants were classified as never or rare drinker $(0 \mathrm{~g} /$ wk), former drinker, light drinker ( $<30 \mathrm{~g} / \mathrm{wk})$, moderate drinker $(30-70 \mathrm{~g} / \mathrm{wk}$ in women and 30-140 g/wk in men) and heavy drinker (>70 g/wk in women and >140 g/wk in men). Physical activity was assessed using the Baecke leisure time physical activity questionnaire [12], and was categorized using tertiles based on the entire cohort.

\section{Exclusions}

It is standard ARIC protocol to exclude African Americans from Washington County, MD or Minneapolis, MN (n=55) and participants who classified their ethnicity as other than White or African American $(n=48)$ because the samples were too small to allow ethnic and center-specific analyses. In addition, we excluded participants who met one of the following conditions: 1) died within one year of visit $1(n=93)$; 2$)$ prevalent CHD or stroke at visit 1 $(\mathrm{n}=950) ; 3)$ missing visit 1 covariates or history of prevalent CHD or stroke $(n=516)$; or 4$)$ missing smoking status or BMI at age $25(n=189)$. This analysis was based on 13,941 White and African American men and women.

\section{Statistical analysis}

Poisson regression was used to calculate mortality rates standardized to the mean value for smoking status at age 25 and age, education, physical activity, alcohol consumption and height at visit 1 . Furthermore, the rates were standardized to a BMI of $21 \mathrm{~kg} / \mathrm{m}^{2}$ and equal distribution by field center. The BMI-mortality relationship was examined on the multiplicative (HR) and additive (hazard difference, HD) scales. The adjusted HRs were calculated using Cox proportional hazard models [13] in SAS version 9.2 (SAS Institute, Inc, Cary, North Carolina). The proportional hazards assumption, tested by examining Kaplan-Meier survival curves and Schoenfeld residuals, was not violated. The adjusted HDs were calculated based on additive risk models [14] in R (R Foundation for Statistical 
Computing, Vienna, Austria). The HRs and HDs were calculated overall and by ethnicgender group.

HRs and HDs per 1,000 person-years for all-cause mortality were calculated using continuous and categorical BMI at age 25. For the categorical analysis, subjects were classified as underweight $\left(<18.5 \mathrm{~kg} / \mathrm{m}^{2}\right)$, normal weight $\left(18.5\right.$ to $\left.<25.0 \mathrm{~kg} / \mathrm{m}^{2}\right)$, overweight (BMI 25.0 to $<30.0 \mathrm{~kg} / \mathrm{m}^{2}$ ) or obese $\left(\geq 30.0 \mathrm{~kg} / \mathrm{m}^{2}\right)$. For the continuous analysis, we chose the most appropriate forms for BMI and weight change by fitting models with quadratic splines. The initial models used quadratic spline regression analysis [15] with 5 knots to examine the shapes of the association of BMI at age 25 and weight change from age 25 to visit 1 with all-cause mortality. More simple models examined BMI and weight change in quadratic or linear forms. Likelihood ratio tests were used to compare nested models.

Separate models using age or follow-up length as the time scale were examined. Results were similar and here we present results from the analyses using follow-up time as the time scale. Because of the large range of ages at visit 1 (45-64 years) the interval over which weight change was assessed varied from 20-39 years. We investigated the impact of this range by comparing results for participants with shorter compared to longer weight change intervals, dividing the study population into two groups with equal numbers of events. We found no differences between the groups in our primary analyses and, therefore, they are combined here. Covariates in the full models included cigarette smoking status at age 25 and age, education, field center, height, cigarette smoking status, alcohol consumption status and physical activity at visit 1 .

\section{RESULTS}

The descriptive characteristics of the analysis sample are shown in Table 1 by ethnic-gender groups. The mean BMI at age 25 was within the normal range for all ethnic-gender groups and slightly higher in men than women. African American women gained an average of 21.5 kg over 20-39 years, which was substantially more than observed in the other groups. For all groups combined, BMI at age 25 and subsequent weight change (age 25 to visit 1) were negatively correlated $(\mathrm{r}=-0.22)$, whereas the correlation between weight change and BMI at visit 1 was positive $(r=0.50)$. The correlation between BMI at age 25 and weight change was null in White women $(r=-0.02, p>0.1)$ and strongest in White men $(r=-0.34)$. Correlations in African American women and men were intermediate at -0.24 and -0.33 respectively. Correlations of BMI at age 25 and BMI at visit 1 ranged from 0.47 (African American women) to 0.59 (White women).

Crude mortality rates were higher in men than women of the same ethnicity and higher in African Americans than Whites of the same gender (Table 2). The rate (per 1,000 personyears) in African American men (19.3, 95\% CI: 17.3-21.2) was over twice that seen in White women (7.5, 95\% CI: 7.0-8.1). Standardizing the rates for age, field center, smoking, education, physical activity, alcohol consumption and height reduced differences between the groups. Standardizing the estimates to a BMI of $21 \mathrm{~kg} / \mathrm{m}^{2}$ at age 25 tended to lower the mortality rates and to further reduce differences among the ethnic-gender groups. Additional standardization for weight change between age 25 and visit 1 had little impact. The fully standardized death rates remained lower in Whites than in African Americans of the same gender, but the differences were smaller than observed in the crude estimates.

Figure 1 shows the adjusted HR from mortality by weight status group, with and without adjustment for weight change. Overall, compared to normal weight adults, the HR was higher in overweight ( $\mathrm{HR}=1.25,95 \% \mathrm{CI}: 1.13-1.38)$ and obese (HR=2.04, 95\% CI: 1.74-2.38) adults. Estimates varied between ethnic and gender groups, but confidence 
intervals were large. Weight change was included in models as a quadratic spline function with 5 knots at the ethnic-gender specific 5th, 15th, 25th, 50th and 95th percentiles. This addition resulted in attenuation of the BMI estimates that was most apparent in obese African American men and women. As shown in Figure 2, patterns of the associations between weight status and mortality examined using differences were similar to those seen using ratios. The categorical analyses shown in Figures 1 and 2 showed generally monotonic associations between BMI and mortality.

For continuous BMI the linear form based on spline analysis and likelihood ratio tests for nested models was appropriate in these data for models that examined HR and those that examined HD. Results from the analyses of continuous BMI are shown in Table 3. The overall mortality HR increased by $28 \%$ for every $5 \mathrm{~kg} / \mathrm{m}^{2}$ increment in BMI at age 25 . After adjusting for weight change, results were attenuated in all ethnic-gender groups, except White men. The confidence interval for the HR included 1.0 in African American men $(\mathrm{HR}=1.05,95 \%$ CI: 0.91-1.22), and there was a statistically significant difference $(\mathrm{p}<0.05)$ between the fully adjusted estimates in African American and White men.

The HD analysis showed that, with all ethnic-gender groups combined, every $5 \mathrm{~kg} / \mathrm{m}^{2}$ increment of BMI was associated with 2.75 (95\% CI: 2.01-3.50) more deaths per 1,000 person-years and this estimate was smaller $(\mathrm{HD}=1.74,95 \% \mathrm{CI}$ : 0.94-2.53) after adjustment for weight change. The effect of BMI on mortality rate difference was significantly higher in African American women compared to White women ( $\mathrm{p}=0.013$ ), but not in African American men compared to White men when weight change was not adjusted. Adjusting for weight change attenuated the results, and BMI was not statistically significant in African American men, but there was no difference between African American and White men detected.

\section{DISCUSSION}

Our analyses showed the estimated impact of young adult BMI on mortality given the subsequent changes in body weight that naturally occurred in this free-living, communitybased sample. Thus, the unadjusted results provided a prediction of the risk of obesity in a similar population of 25 year old adults, assuming that body weight would change with aging as occurred in our free-living cohort. We found that elevated BMI at age 25 was associated with more deaths than being normal weight at age 25 , and the finding was consistent in the overall and the ethnic-gender specific analyses and was seen in both HR and HD.

Our results indicated that associations between BMI and mortality were as strong in African Americans as in Whites and perhaps even slightly stronger in African American women than White women when assessed using risk difference. This finding contrasts with some previous studies showing weaker associations in African American compared to White women [16]. The Cancer Prevention Study II (CPS-II) cohort, for example, included participants aged 30-111 years and used recent information (baseline in 1982) and a geographically diverse sample while controlling for possible confounding by smoking and pre-existing illness [17, 18]. Despite a large sample size and good statistical power, no statistically significant associations were found between elevated BMI and all-cause mortality in African Americans but elevated BMI increased mortality in Whites.

In a review on the effect of BMI on all-cause mortality in African Americans in 2000 [16], it was noted that several studies indicated that the impact of BMI on mortality may be weaker in African Americans compared to Whites. Since the appearance of this review, we know of only three studies that have compared associations of BMI and all-cause mortality between 
African Americans and White Americans [19-21]. Interactions with ethnicity and BMI were not always tested and confidence intervals were large or not shown, but nevertheless, all three studies showed that the relative risk associated with elevated BMI in African Americans tended to be smaller than that observed in White Americans.

One concern about the interpretation of these studies stems from the use of risk estimates in the form of ratios (odds ratios or HR) [22]. Often missing in the discussion is an appreciation of the potential impact of differences in the incidence of the outcome at the reference level of the exposure (e.g. death rates in normal weight African Americans compared to normal weight Whites). When these levels are substantially different, comparisons between ethnic groups can be in opposite directions depending on whether a ratio or a difference is used to estimate effects [22]. In these instances we have suggested investigators examine both risk ratios and risk differences and compare the trends between groups cautiously. Since death rates are higher in African Americans than in Whites [23], the type of estimates compared could be important. In an analysis comparing the BMImortality associations, higher death rates among the normal weight could result in lower risk ratios in obese African Americans (calculated as the death rate in obese divided by the death rate in normal weight), even when the difference in the number of deaths (calculated as the death rate in obese minus the death rate in normal weight) was larger in African Americans compared to Whites.

In our analyses of ethnic and gender groups combined, adjusting for weight change from age 25 to visit 1 only slightly attenuated the HR and HD. This result suggested that BMI at age 25 may influence mortality even when subsequent weight change was controlled and that the impact of obesity in young adults was not dependent on subsequent changes in BMI. This overall finding was not seen in the separate analyses of African American men among whom BMI was not associated with mortality after adjustment for weight change. Compared to the other groups studied here, the association of BMI at age 25 with mortality in African American men was apparently more dependent on weight changes, although the correlation between BMI at age 25 and weight change $(r=-0.33)$ was not larger than in other groups. Weight change could be serving as a marker for important lifestyle factors in African American men that were not measured here. To our knowledge, no other study has investigated the link between long-term weight change and mortality in an African American sample.

Estimates from previous studies on BMI in early adulthood and mortality among predominantly Whites were consistent with our risk ratios [5-8]; Yarnell et al. (2000) [7], for example, reported estimates of 1.25 (95\% CI: 1.13, 1.38) for overweight and 2.04 (95\% CI: $1.74,2.38$ ) for obese participants in the analysis without adjustment for weight change.

Of the six studies examining BMI in early adulthood that we mentioned previously [5-10], only two used measured body weight in young adulthood [5, 8]. One examined 629 men in the Glasgow Alumni Cohort whose body weight was measured at the Student Health Service between 1948 and 1949 when the men were at a median age of 22 years [5]. The second used data from 79,657 Dutch men aged 18 years from compulsory military medical examinations [8]. Both studies found estimates similar to those found here, which is reassuring given the fact that our data on weight at age 25 was self-reported and was recalled over an interval of 20 years or longer. Self-reported weight is prone to measurement error, yet it does provide a reasonable proxy of measured weight with correlation coefficients above 0.9 for concurrent estimates [24]. In addition, it has been shown that recall of weight much earlier in life (28 years prior) is also highly correlated (0.82) with weight measured at that time. Nevertheless, there is bias toward the mean, and 
categorization of participants into weight status groups dependent upon absolute BMI is imperfect [25].

It was a limitation of our study that the samples were not nationally representative and that ethnicity was partially confounded by geographic location since African Americans were studied in only two, and Whites in only three, of the four study centers. This study did not include any deaths in individuals who died before the age of 45 . It has been shown that obesity-associated risk ratios tend to be larger in younger individuals compared to older, whereas positive associations with age are seen when risk differences are examined [16]. Since patterns of BMI associations were similar in this study in both the ratio and the difference analyses, it is unlikely that this feature of our study design seriously biased our results, but we cannot know this with certainty. It was a strength of this work that we examined risk using both ratios and differences. We hypothesized that higher levels of mortality in normal weight African Americans compared to Whites might result in BMI associated risk ratios being lower and risk differences being similar in African Americans compared to Whites. However, similar levels of risk were observed using ratios and larger risk using differences for African American (4.33, 95\% CI: 2.47, 6.19) compared to White women $(1.65,95 \% \mathrm{CI}: 0.65,2.64)$ in analyses without control for weight change. The greater number of deaths associated with obesity in young adult African American women compared to White women, shown here in our analysis of risk differences, is a novel finding. Since the number of deaths is more directly linked to public health impact, this could indicate that obesity is more detrimental in African American women. In African American and White men, both risk ratios and risk differences were similar.

In African American men, BMI was null in models including weight change, while associations were maintained or only mildly attenuated in other ethnic-gender groups. Causes of death in African Americans have been shown to differ from those of Whites [26]. These differences are most dramatic in African American men who suffer higher rates of death from causes that are not likely linked to obesity, such as assault and homicide, viral infections including HIV and viral hepatitis, septicemia, and pneumonia [23]. Differences in the spectrum of causes may make associations between obesity and all-cause mortality less clear in African American men. More work is needed to better understand the impact of obesity on mortality in African American populations.

\section{Acknowledgments}

The Atherosclerosis Risk in Communities Study is carried out as a collaborative study supported by National Heart, Lung, and Blood Institute contracts N01-HC-55015, N01-HC-55016, N01-HC55018, N01-HC-55019, N01HC-55020, N01-HC-55021, N01-HC-55022 and 1RC1HL099429. The authors thank the staff and participants of the ARIC study for their important contributions.

\section{References}

1. Centers for Disease Control and Prevention (CDC), National Center for Health Statistics (NCHS). National Health and Nutrition Examination Survey Questionnaire III. Services DoHaH; Hyattsville, MD: 1988-1994.

2. Centers for Disease Control and Prevention (CDC), National Center for Health Statistics (NCHS). National Health and Nutrition Examination Survey Questionnaire III. Services DoHaH; Hyattsville, MD: 1999-2004.

3. Flegal K, Carroll M, Ogden C, et al. Prevalence and trends in obesity among US adults, 1999-2000. JAMA. 2002; 288:1723-1727. [PubMed: 12365955]

4. Flegal KM, Carroll MD, Ogden CL, et al. Prevalence and trends in obesity among US adults, 1999-2008. JAMA. 2010; 303:235-241. [PubMed: 20071471] 
5. Jeffreys M, McCarron P, Gunnell D, et al. Body mass index in early and mid-adulthood, and subsequent mortality: a historical cohort study. Int J Obes. 2003; 27:1391-1397.

6. Corrada MM, Kawas CH, Mozaffar F, et al. Association of body mass index and weight change with all-cause mortality in the elderly. Am J Epidemiol. 2006; 163:938-949. [PubMed: 16641311]

7. Yarnell JW, Patterson CC, Thomas HF, et al. Comparison of weight in middle age, weight at 18 years, and weight change between, in predicting subsequent 14 year mortality and coronary events: Caerphilly Prospective Study. J Epidemiol Community Health. 2000; 54:344-348. [PubMed: 10814654]

8. Hoffmans M. The impact of body mass index of 78,612 18-year old Dutch men on 32-year mortality from all causes. J Clin Epidemiol. 1988; 41:749-756. [PubMed: 3418364]

9. Shimazu T, Kuriyama S, Ohmori-Matsuda K, et al. Increase in body mass index category since age 20 years and all-cause mortality: a prospective cohort study (the Ohsaki Study). Int J Obes (Lond). 2009; 33:490-496. [PubMed: 19223845]

10. Rhoads G, Kagan A. The relation of coronary disease, stroke, and mortality to weight in youth and in middle age. Lancet. 1983; 1:492-495. [PubMed: 6131209]

11. Investigators A. The Atherosclerosis Risk in Communities (ARIC) Study: Design and objectives. Am J Epidemiol. 1989; 129:687-702. [PubMed: 2646917]

12. Baecke J, Burema J, Frijters J. A short questionnaire for the measurement of habitual physical activity in epidemiological studies. Am J Clin Nutr. 1982; 36:936-942. [PubMed: 7137077]

13. Cox D. Regression models and life tables. J R Stat Soc. 1972; 34:457-481.

14. Lin DY, Ying Z. Semiparametric analysis of the additive risk model. Biometrika. 1994; 81:61-71.

15. Witte J, Greenland S. A nested approach to evaluating dose-response and trend. Ann Epidemiol. 1997; 7:188-193. [PubMed: 9141641]

16. Stevens J. Obesity and mortality in African Americans. Nutr Rev. 2000; 58:346-358. [PubMed: 11140906]

17. Calle E, Thun M, Petrelli J, et al. Body-mass index and mortality in a prospective cohort of U.S. adults. N Engl J Med. 1999; 341:1097-1105. [PubMed: 10511607]

18. Calle EE, Rodriguez C, Jacobs EJ, et al. The American Cancer Society Cancer Prevention Study II Nutrition Cohort: rationale, study design, and baseline characteristics. Cancer. 2002; 94:24902501. [PubMed: 12015775]

19. Sanchez A, Reed D, Price R. Reduced mortality associated with body mass index (BMI) in African Americans relative to Caucasians. Ethn Dis. 2000; 10:24-30. [PubMed: 10764127]

20. Livingston EH, Ko CY. Effect of diabetes and hypertension on obesity-related mortality. Surgery. 2005; 137:16-25. [PubMed: 15614276]

21. Reis JP, Araneta MR, Wingard DL, et al. Overall obesity and abdominal adiposity as predictors of mortality in U.S. White and black adults. Ann Epidemiol. 2009; 19:134-142. [PubMed: 19185808]

22. Stevens J, Juhaeri, Cai J, et al. The effect of decision rules on the choice of a body mass index cutoff for obesity: examples from African American and white women. Am J Clin Nutr. 2002; 75:986-992. [PubMed: 12036803]

23. Heron M, Hoyert DL, Murphy SL, et al. Deaths: final data for 2006. Natl Vital Stat Rep. 2009; 57:1-134. [PubMed: 19788058]

24. Stevens J, Keil J, Waid R, et al. Accuracy of current, 4-year, and 28-year self-reported body weight in an elderly population. Am J Epidemiol. 1990; 132:1156-1163. [PubMed: 2260547]

25. Plankey M, Stevens J, Flegal K, et al. Prediction equations do not eliminate systematic error in self-reported body mass index. Obes Res. 1997; 5:308-314. [PubMed: 9285836]

26. Heron M. Deaths: leading causes for 2006. Natl Vital Stat Rep. 2010; 58:1-100. [PubMed: 20361522] 


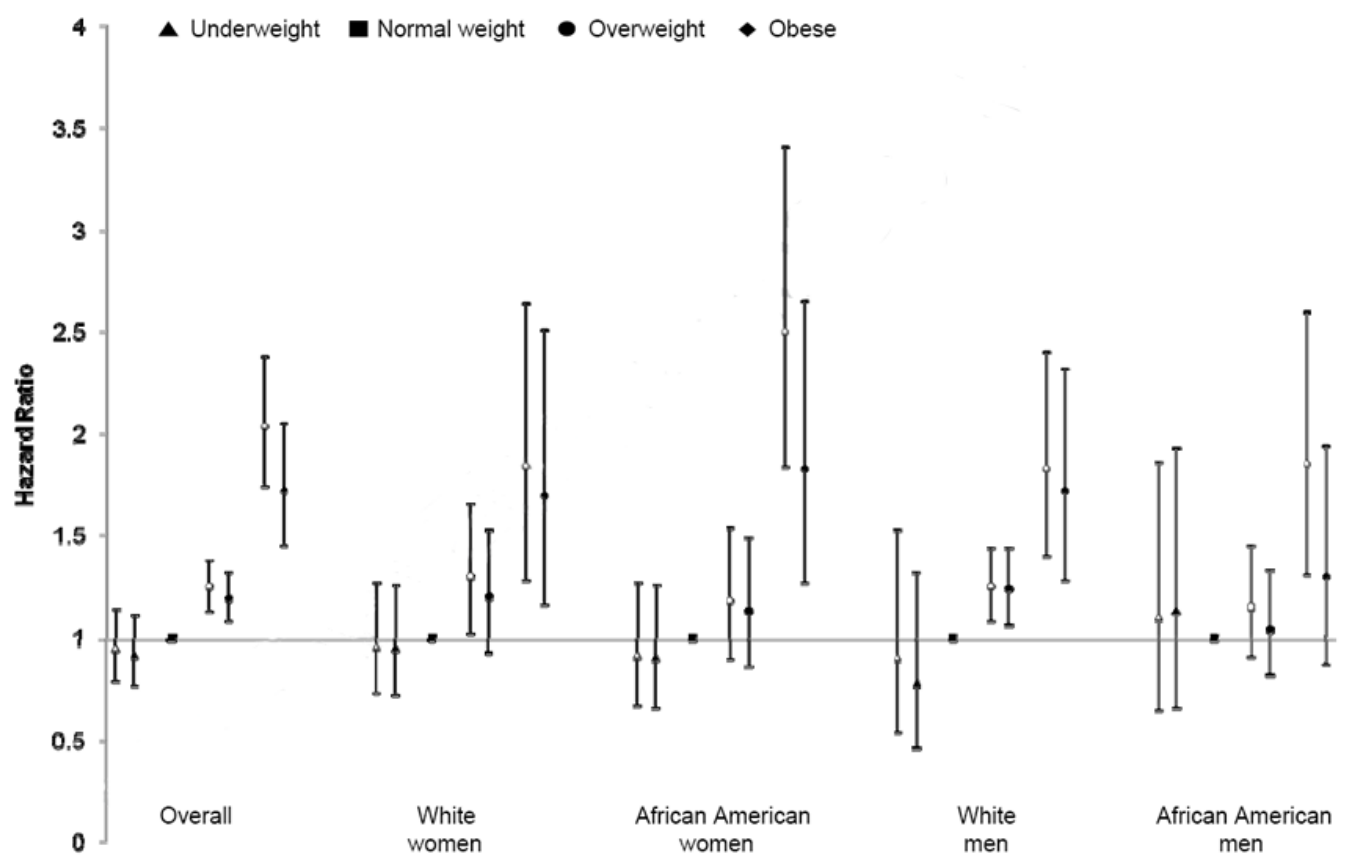

Figure 1.

Adjusted hazard ratios for mortality by weight status category (at age 25) overall and by ethnic-gender group. Models were adjusted for smoking status at age 25 and age, education, field center, smoking status, physical activity, alcohol consumption and height at visit 1 . Overall model also adjusted for gender and race. Open symbols are from models not adjusted for weight change. Closed symbols are from models adjusted for weight change (measured weight at visit 1 minus self-reported weight at age 25 and modeled using a quadratic spline function with 5 knots). 


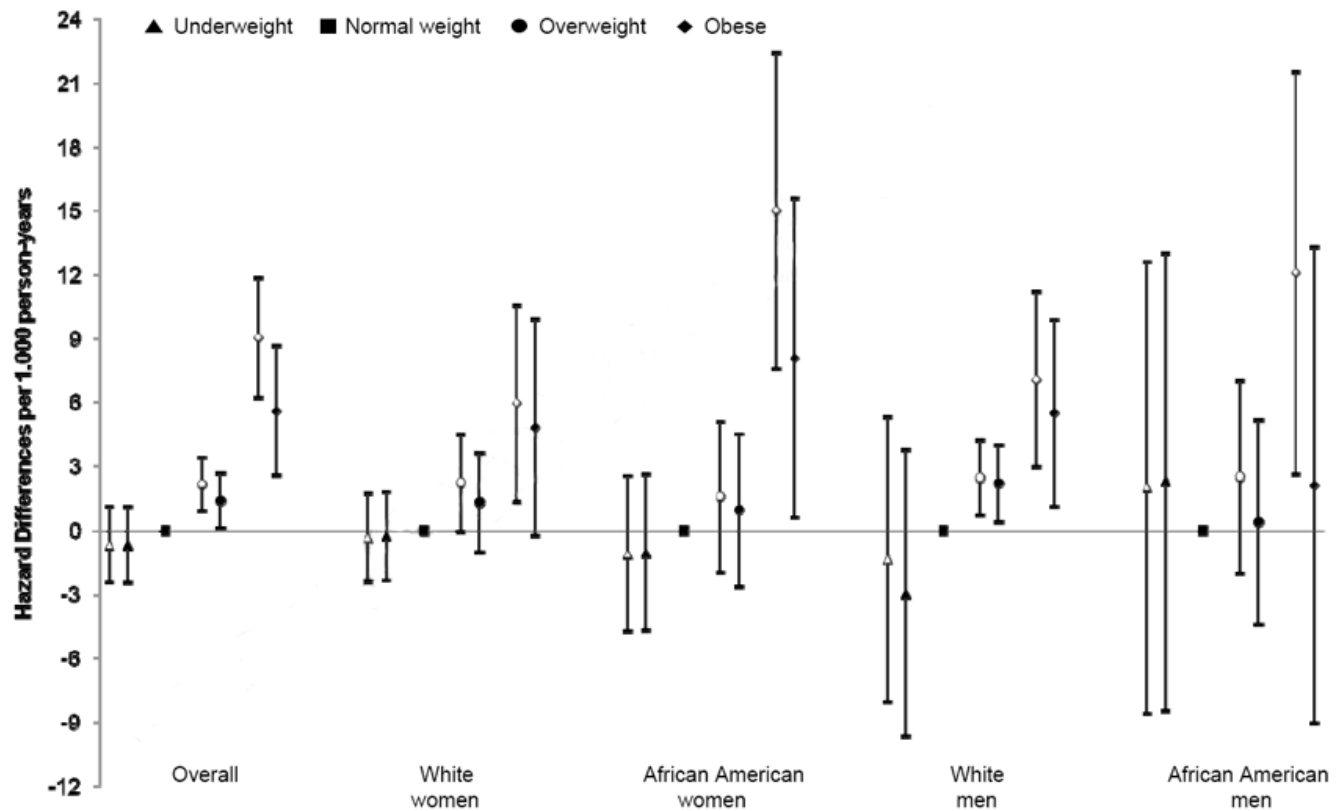

Figure 2.

Adjusted hazard differences per 1,000 person-years for mortality by weight status category (at age 25) overall and by ethnic-gender group. Models were adjusted for smoking status at age 25 and age, education, field center, smoking status, physical activity, alcohol consumption and height at visit 1 . Overall model also adjusted for gender and race. Open symbols are from models not adjusted for weight change. Closed symbols are from models adjusted for weight change (measured weight at visit 1 minus self-reported weight at age 25 and modeled using a quadratic spline function with 5 knots). 
Table 1

Descriptive characteristics of the analysis sample by ethnic-gender groups

\begin{tabular}{|c|c|c|c|c|}
\hline & White women $(\mathbf{n = 5 , 6 5 2})$ & $\begin{array}{l}\text { African American } \\
\text { women }(n=2,272)\end{array}$ & White men $(n=4,693)$ & $\begin{array}{c}\text { African American } \\
\text { men }(n=1,324)\end{array}$ \\
\hline Age at visit 1 (years, mean (SD)) & $53.9(5.7)$ & $53.2(5.7)$ & $54.5(5.7)$ & $53.5(5.9)$ \\
\hline \multicolumn{5}{|l|}{ BMI at age $25\left(\mathrm{~kg} / \mathrm{m}^{2}, \%\right)$} \\
\hline Underweight & 8.1 & 10.6 & 1.5 & 3.7 \\
\hline Normal & 80.6 & 69.5 & 62.6 & 63.7 \\
\hline Overweight & 8.6 & 14.0 & 30.6 & 25.5 \\
\hline Obese & 2.7 & 5.9 & 5.3 & 7.1 \\
\hline Weight at age $25\left(\mathrm{~kg}^{a}\right.$, mean (SD)) & $56.9(9.0)$ & $59.4(11.3)$ & $74.6(11.5)$ & $74.0(13.1)$ \\
\hline Weight change ${ }^{b}(\mathrm{~kg} a$, mean (SD)) & $12.2(11.5)$ & $21.5(15.6)$ & $9.8(10.9)$ & $10.9(14.1)$ \\
\hline Height (cm, mean (SD)) & $162.0(5.9)$ & $163.1(6.1)$ & $176.3(6.5)$ & $176.1(6.7)$ \\
\hline \multicolumn{5}{|l|}{ Education (\%) } \\
\hline Less than high school & 15.7 & 38.8 & 16.3 & 41.2 \\
\hline High school or equivalent & 51.0 & 29.4 & 39.2 & 26.4 \\
\hline At least some college & 33.3 & 31.7 & 44.5 & 32.4 \\
\hline Cigarette smokers at age $25(\%)$ & 40.7 & 33.2 & 64.6 & 65.3 \\
\hline \multicolumn{5}{|l|}{ Smoking status at visit $1(\%)$} \\
\hline Never & 51.0 & 58.8 & 29.4 & 29.6 \\
\hline Former & 24.4 & 17.3 & 46.4 & 32.6 \\
\hline Current & 24.6 & 23.8 & 24.1 & 37.8 \\
\hline \multicolumn{5}{|l|}{ Alcohol consumption at visit $1(\%)$} \\
\hline Never/rare & 52.2 & 65.1 & 25.5 & 27.6 \\
\hline Former & 13.7 & 19.7 & 19.3 & 26.6 \\
\hline Light & 10.9 & 4.8 & 11.0 & 6.4 \\
\hline Moderate & 10.8 & 5.1 & 26.1 & 23.0 \\
\hline Heavy & 12.3 & 5.4 & 18.1 & 16.3 \\
\hline \multicolumn{5}{|l|}{ Physical activity at visit $1(\%)$} \\
\hline 1st tertile $(1.00-2.00)$ & 40.2 & 61.2 & 30.0 & 52.6 \\
\hline 2nd tertile (2.01-2.75) & 33.1 & 26.0 & 30.3 & 27.6 \\
\hline 3rd tertile $(2.76-5.00)$ & 26.7 & 12.9 & 39.7 & 19.9 \\
\hline
\end{tabular}

$a_{1 \mathrm{lb}=0.45 \mathrm{~kg} ;}$

$b_{\text {Weight change calculated as measured weight at visit } 1 \text { minus self-reported weight at age } 25}$ 


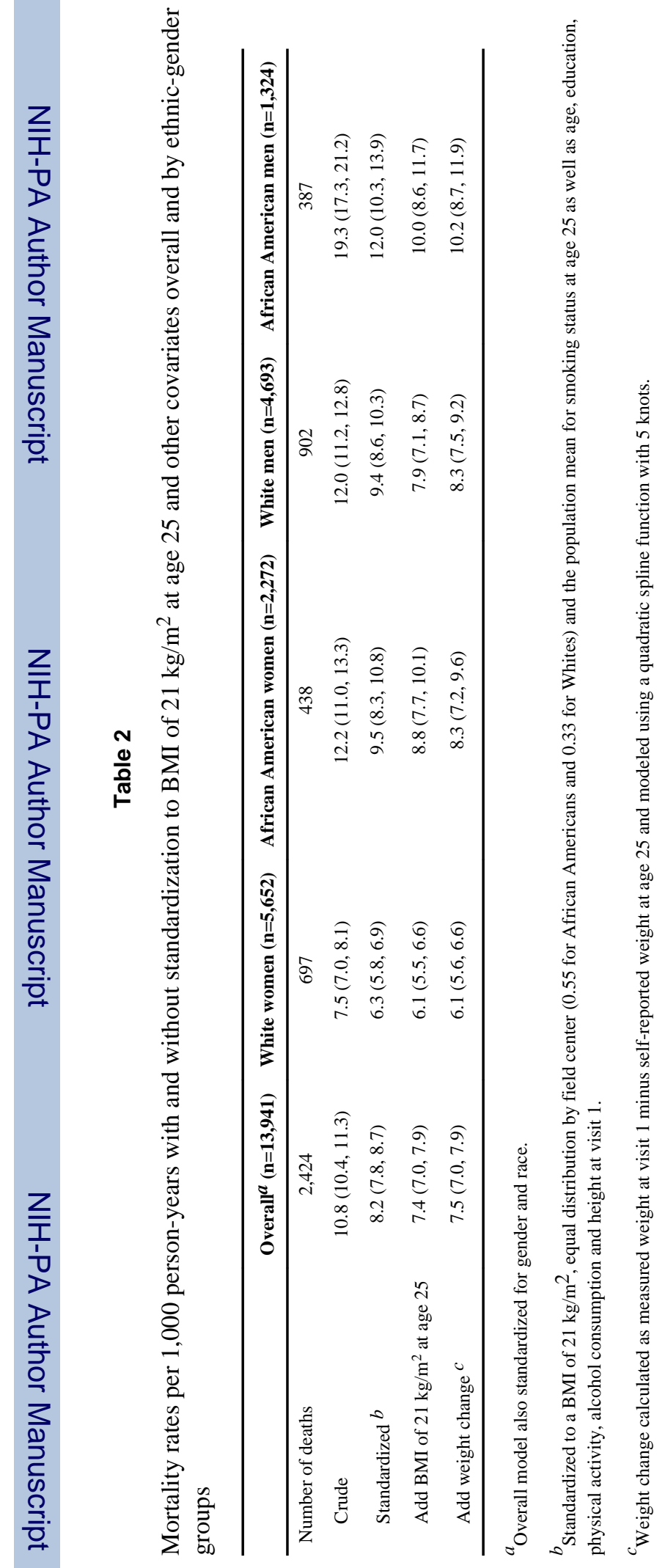




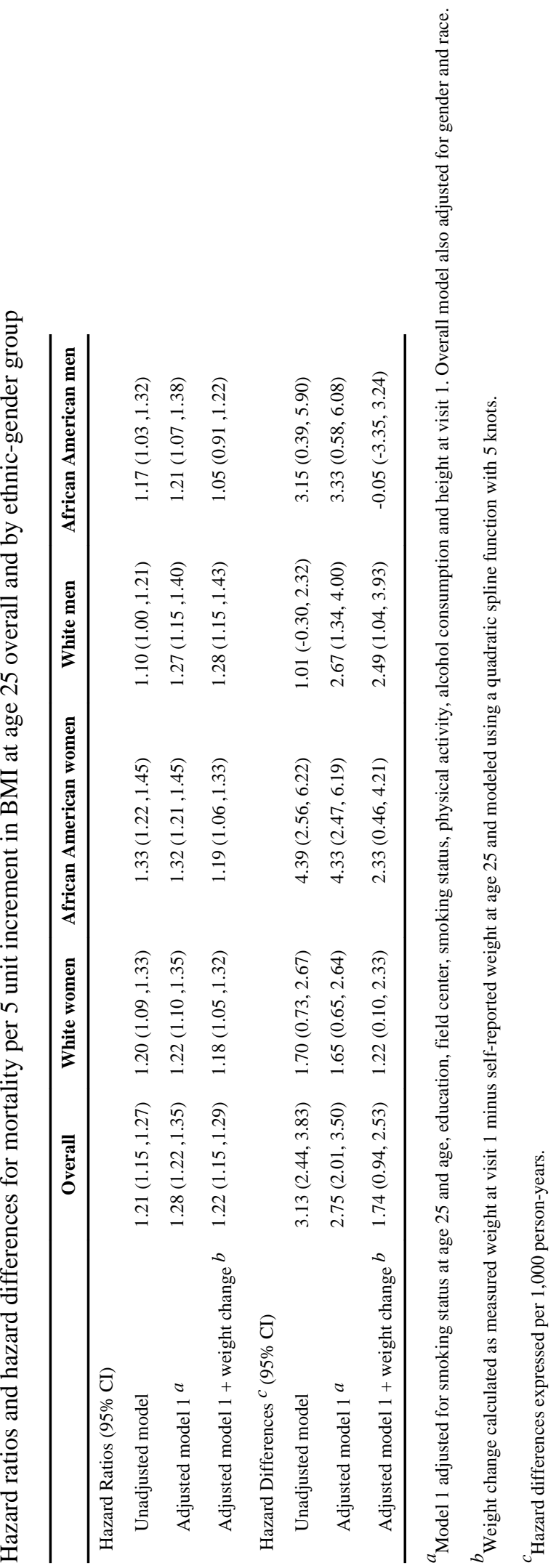

